\section{The science and myths of smell}

Tim Jacob

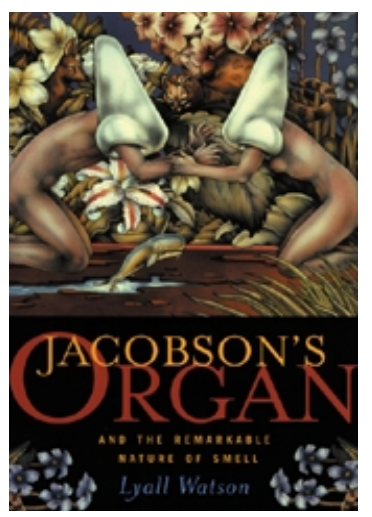

Jacobson's Organ and the Remarkable Nature of Smell

by Lyall Watson

W.W. Norton \& Co., New York, NY

255 pages, US\$ 24.95

ISBN 0393049086

The sense of smell presents us with a conundrum. While we find certain smells pleasing, for example the scents of flowers, we often object to human odours and spend a great deal of effort and money disguising our own smell. This delightful book by Irish naturalist and author Lyall Watson investigates the role of smell in the animal and plant kingdoms and attempts to unravel its mysteries. During the course of this adventure, we are taken to some exotic locations and witness some extraordinary examples of animal and plant behaviour.

There are, for example, the Ongee people of the Andaman Islands who elevate the sense of smell to a cosmic principle, believing that odour is the source of personality and that their spirit resides in the nose. We are told that some Mexican men still believe that their breath odour is more responsible for conception than their semen. Then there are the mule deer that deliberately urinate down their legs to soak the tufts of hair over the tarsal gland and then rub their hinds legs together in a mincing manner, like a group of women in high heels and tight skirts. This ritual is performed after the herd has been threatened and it seems to have a settling effect enhancing group cohesion. Watson also describes the curious Lophiomys, a rodent that resembles a cross between a possum and a badger, which has two belts of glands and specialised hairs along each side of its body. When alarmed, these glands secrete a chemical that has the effect of making any human or animal in the vicinity feel dry-mouthed and uneasy. In a world where creatures this size tend to form part of another's diet, Lophiomys is often left alone.

Chemical communication is not confined to the animal kingdom since plants do some pretty strange things too. Watson describes how the acacia tree responds to browsing, or being beaten with a stick, by increasing the levels of tannin in its leaves within minutes. Remarkably, the tannin levels then rise in neighbouring trees, and, due to its bitter taste, repel the browsers before they can do any further damage. This example of plant communication is not restricted to acacia trees, and has been demonstrated in other plants as well as between plants and animals. Tearing the leaves of seedling poplar trees results in a widespread increase in phenols, which are known to inhibit the growth of butterfly larvae. Tobacco plants warn each other of tobacco mosaic virus attack by releasing methyl salicylate, which is then converted to the protective salicylic acid in uninfected plants.

It is this subject, chemical communication, whether between plants or animals, that is the central concern of this book. Watson invokes the idea of an 'odornet', a general and universal system of chemical communication in which all life, both plant and animal, is involved. The odornet results in a coordinated ecosystem where everything knows its place-if only this were true!

This book is a heady mixture of hard science and mythology - a combination that at times feels like a confidence trick.
The facts/smells come in thick and fast until your resistance/senses are overwhelmed. The key player in this odorous universe is a little known organ named after its discoverer, Jacobson; also known as the vomeronasal organ. It mediates pheromonal communication in some animals, while it is absent in others, e.g. birds. The organ is definitely present in humans-one little characteristic pit on the floor of each nostril by the nasal septum about $1.5 \mathrm{~cm}$ in from the margin - but the question is, and here is the crunch, are they functional? Watson gives us the usual evidence of menstrual synchrony in women and the strange coincidences and unexplained feelings that sometimes accompany smell. He delves into the relationship between smell and sex, and finds that 'human pheromones' have been isolated, identified-they are androstadienone and estratetraenoland added to perfume. The company that has done this has also funded research that demonstrates that these compounds activate the Jacobson's organ and, much more controversially, the limbic system of the brain. This is an area where most biologists and zoologists tread with caution since it is littered with unsubstantiated and outrageous claims for aphrodisiacs. But, of course, Watson's whole story would fall rather flat if he didn't present us with a functional mechanism. However, the evidence is slight and the consensus in the olfactory community is that the Jacobson's organ ceases to be functional soon after birth. Does this detract from the book? Not at all. It is a wonderful compendium of smell facts and myths and even if the Jacobson's organ does not function in adult humans, menstrual synchrony occurs without doubt, and we have all experienced smellinduced déjà vu. The fact that, in humans, these phenomena may be mediated by the more conventional olfactory organ does not make them any less remarkable.

The author is at the School of Biosciences, Cardiff University, Cardiff, UK.

DOI: $10.1093 / \mathrm{embo-reports/kve220}$ 